\title{
Airway compression after arthroscopic shoulder surgery under general anesthesia
}

\author{
Min-Young No, Pyoung-On Kim, and Won-Jun Choi \\ Department of Anesthesiology and Pain Medicine, Sungkyunkwan University School of Medicine, Kangbuk Samsung Hospital, \\ Seoul, Korea
}

Arthroscopic surgery is less invasive and has more visibility into the details of anatomical structures compared to open techniques. With the advantages of less pain and quicker rehabilitation, its popularity is rising [1]. Despite such advantages, the complication incidence rate is $2-5 \%$. Among these complications, airway compression-related cases have been reported, which are rare but worthy of anesthesiologists' attention $[1,2]$. We also experienced airway obstruction.

A 66-year-old woman was to undergo arthroscopic acromioplasty and rotator cuff repair. The patient had no medical history, except hypertension, and was overweight with a body mass index of $27.39 \mathrm{~kg} / \mathrm{m}^{2}$. There was no abnormality on preoperative blood test, chest X-ray (CXR) and electrocardiogram. Categorized as ASA class II and Mallampati class II, she had a relatively short neck with sternomental distance of $10 \mathrm{~cm}$.

Prior to general anesthesia, interscalene nerve block (ISB) was performed for postoperative analgesia. Complications such as dyspnea were not detected. Anesthesia was induced with propofol and rocuronium. Afterwards, we made an endotracheal intubation under direct laryngoscope. We classified the airway as class II by Cormack-Lehane classification. Anesthesia was maintained with desflurane $3-5 \mathrm{vol} \%$ in $50 \% \mathrm{O}_{2}$. The patient was placed in the right lateral decubitus. Tearing of the supraspinatus muscle was sutured and acromioplasty was performed. A total of $15,000 \mathrm{cc}$ of normal saline was infused by a pressure pump at the pressure of $60-80 \mathrm{mmHg}$ for 120 minutes into the surgical space. Intraoperative vital signs remained stable. The operation and anesthesia time were 140 minutes and 185 minutes, respectively.
After the operation, the patient was repositioned to the supine and was extubated. The vital signs after arriving to the recovery room were blood pressure of $117 / 63 \mathrm{mmHg}$ and a heart rate of 71 beats/min, similar to those after extubation. However, she abruptly complained of breast discomfort and severe dyspnea while oxygen saturation $\left(\mathrm{SpO}_{2}\right)$ decreased to $90 \%$. Suspecting phrenic nerve palsy caused by ISB, we tried to calm her by providing oxygen, but the symptoms did not change. Therefore, she was placed in a sitting position along with a head tilt-chin lift position and positive pressure ventilation for spontaneous breathing by a non-rebreathing circuit and prepared intubation was performed. In 2 minutes, $\mathrm{SpO}_{2}$ returned to $97 \%$, but she still complained of dyspnea. We conducted a blood gas analysis (ABGA), auscultation and CXR. When we removed her top and velpeau arm sling for auscultation, widespread edema accompanied with tenderness was observed from the neck to the chest (Fig. 1). On auscultation, left lung sound decreased. ABGA was $\mathrm{PH} 7.34, \mathrm{PaCO}_{2}$ $40 \mathrm{mmHg}, \mathrm{PaO}_{2} 411 \mathrm{mmHg}, \mathrm{HCO}_{3}^{-} 21 \mathrm{mEq} / \mathrm{L}$ and $\mathrm{SaO}_{2} 100 \%$. CXR revealed a subcutaneous fluid collection in the left chest and displacement of the airway to the right (Fig. 1). We decided to observe her while keeping her in the sitting position and providing $\mathrm{O}_{2}$ at $5 \mathrm{~L} / \mathrm{min}$. $\mathrm{SpO}_{2}$ was maintained at $98-100 \%$ for two hours, and then the patient was moved to the general ward with the oxygen mask.

On shoulder arthroscopy, fluid continuously flushed into the joints to secure more visibility of the intra-articular space, when extravasation may occur. In most cases, fluid-induced extravasation is reabsorbed within 12 hours and is clinically not

Corresponding author: Won-Jun Choi, M.D., Department of Anesthesiology and Pain Medicine, Sungkyunkwan University School of Medicine, Kangbuk Samsung Hospital, 108, Pyeong-dong, Jongno-gu, Seoul 110-746, Korea. Tel: 82-2-2001-2001, Fax: 82-2-2001-2326, E-mail: wj2006. choi@samsung.com

(C) This is an open-access article distributed under the terms of the Creative Commons Attribution Non-Commercial License (http:// creativecommons.org/licenses/by-nc/3.0/), which permits unrestricted non-commercial use, distribution, and reproduction in any medium, provided the original work is properly cited. 

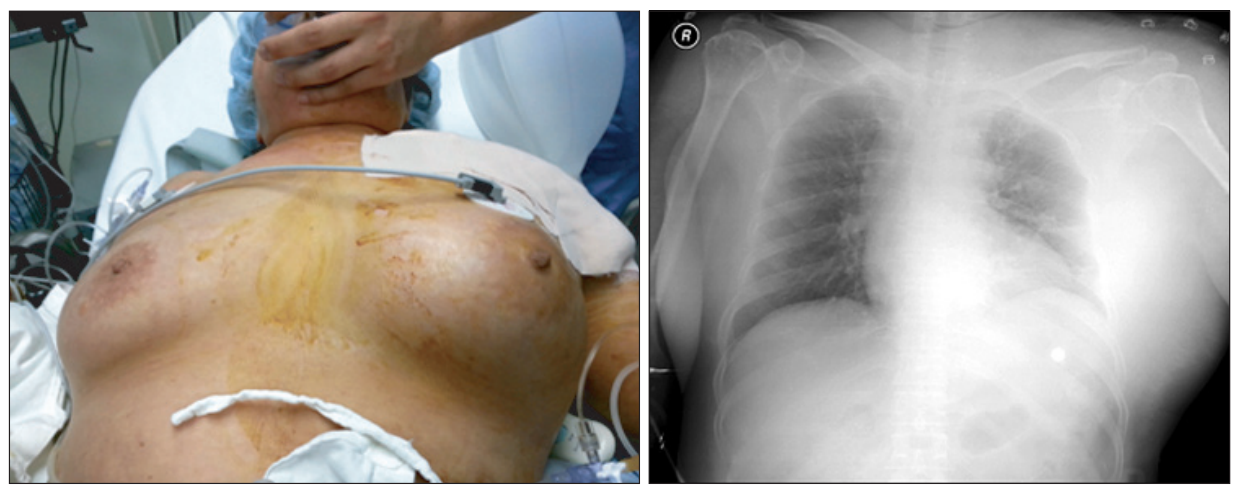

Fig. 1. Upper airway compromise by extravasated fluid: a rare complication after arthroscopic repair of atrophic cuff tear.

problematic [2]. However, the extra-articular leakage may cause upper airway obstruction. The risk factors of massive extravasation are high pump pressure, obesity, lengthy operation time and subacromial arthroscopic surgery [1]. Owing to adipose layers, obese patients are more vulnerable to the extravasation of irrigation fluid through the periarticular soft tissues because subcutaneous fat layers are extensively dispersed. For elderly patients, the diffusion of irrigation fluid occurs more because of the looseness in the skin and subcutaneous tissue [3]. For unencapsulated subacromial space, the extravasation of fluid occurs more frequently during acromicroplasty. In patients with damaged supraspinatus muscle or the occurrence of iatrogenic lesions on the deltoid muscle, hypodermic edema occurs more often [2]. The lateral position also may cause subcutaneous fluid to spread from the shoulder to the neck by gravity [3].

As the causing factors in this case, we counted in the relatively long operation time, subacromial arthroscopy, obesity, old age, right lateral decubitus, and damaged supraspinatus muscle. However, we neglected the possibility of airway compression induced by extravasation of irrigation fluid due to the following reasons: first, when the patient complained of dyspnea, we thought of phrenic nerve block by ISB. Second, it was difficult to discern the airway compression during operation since the neck and the chest were covered with a surgical drap. Third, she was overweight and had a short neck. Therefore, we could not pre- cisely guess how big the edema grew, compared with the preoperative state. Moreover, the surgical site was covered with several layers of gauze and pad, on top of which a velpeau arm sling was worn, which made it difficult to observe the patient.

Previous case reports raised the awareness of the possibility of intraoperative airway obstruction [1-3]; however, it is possible for airway obstruction to occur not only in the operation room, but also in the recovery room, as in our case. It is fathomed to be related to the postoperative posture alteration from the lateral decubitus position to the supine. The supine position is higher in airway collapsibility than in the lateral position [4]. When the patient was repositioned to the supine position, the edema-induced swollen anterior pharyngeal structure was likely to droop downward due to gravity, which may press harder on the already narrowed upper airway, leading to dyspnea.

For an anesthesiologist who is to make a precise judgment and take prompt action in a situation such as this case, it is important to be well acquainted with the risk factors of extravasation during shoulder arthroscopic surgery. When patients with such factors are to undergo arthroscopic surgery, it is advised to keep close observation on the development of edema by taking frequent checks and tactile perceptions on the neck and the chest. Also, if patients complain of dyspnea, it would be helpful to bear the possibility of irrigation fluid-induced airway compression in mind.

\section{References}

1. Venkat G, Moon YL, Na WC, So KY. Upper airway compromise by extravasated fluid: a rare complication after arthroscopic repair of atrophic cuff tear. Orthopedics 2009; 32: 776-8.

2. Borgeat A, Bird P, Ekatodramis G, Dumont C. Tracheal compression caused by periarticular fluid accumulation: a rare complication of shoulder surgery. J Shoulder Elbow Surg 2000; 9: 443-5.

3. Yoshimura E, Tano T, Ichinose K, Ushijima K. Airway obstruction involving a laryngeal mask airway during arthroscopic shoulder surgery. J Anesth 2005; 19: 325-7.

4. Walsh JH, Leigh MS, Paduch A, Maddison KJ, Armstrong JJ, Sampson DD, et al. Effect of body posture on pharyngeal shape and size in adults with and without obstructive sleep apnea. Sleep 2008; 31: 1543-9. 